\title{
The laminar-turbulent transition in a fibre laser
}

E.G. Turitsyna ${ }^{1}$, S.V. Smirnov ${ }^{2}$, S. Sugavanam ${ }^{1}$, N. Tarasov ${ }^{1}$, X. Shu ${ }^{1}$, S.A. Babin ${ }^{2,3}$, E.V. Podivilov ${ }^{2,3}$, D.V. Churkin ${ }^{1,2,3}$, G. Falkovich ${ }^{4}$, and S. K. Turitsyn ${ }^{1,2, *}$

${ }^{1}$ Aston Institute of Photonic Technologies, Aston University, Birmingham, B4 7ET, UK

${ }^{2}$ Novosibirsk State University, Novosibirsk, 630090 Russia

${ }^{3}$ Institute of Automation and Electrometry, Siberian Branch, Russian Academy of Sciences, Novosibirsk, 630090, Russia

${ }^{4}$ Weizmann Institute of Science, Rehovot 76100 Israel and Institute for Information Transmission Problems, Moscow, 127994 Russia

Studying the transition from a linearly stable coherent laminar state to a highly disordered state of turbulence is conceptually and technically challenging and of great interest since all pipe and channel flows are of that type $\mathrm{e}^{1,2}$. In optics, understanding how a system loses coherence, as spatial size or the strength of excitation increases, is a fundamental problem of practical importance ${ }^{3-5}$. Here we report our studies of a fibre laser that operates in both laminar and turbulent regimes. We show that the laminar phase is analogous to a one-dimensional coherent condensate and the onset of turbulence is due to the loss of spatial coherence. Our investigations suggest that the laminar-turbulent transition in the laser is due to condensate destruction by clustering dark and grey solitons. This finding could prove valuable for the design of coherent optical devices as well as systems operating far from thermodynamic equilibrium. 
Nature does not allow us to increase the size of a system without eventually losing coherence. For example, even though a coherent laminar flow through a pipe is always linearly stable, increasing the pipe diameter or speed eventually makes the flow turbulent, which vastly increases $\operatorname{drag}^{1,2,6-9}$. One needs to identify the origins of the onset of turbulence to understand and control it. It is a formidable task for a linearly stable system, due to the lack of a linear instability analysis, which shows what destroys the laminar state and helps identify the patterns that appear instead ${ }^{2,6-9}$.

In optical fibres with normal dispersion, a coherent monochromatic wave or spectrally narrow packets are linearly stable with respect to modulation instability ${ }^{10}$. In a laser cavity with normal dispersion, it is theoretically possible to overcome wave dephasing by nonlinear four-wave-mixing and achieve a classical wave condensation forming a coherent state ${ }^{11,12}$. A kinetic condensation of classical waves was recently observed in two-dimensional, optical Hamiltonian systems, making a link with the BoseEinstein condensation, including condensation of photons ${ }^{13,14}$. However, operational regimes in many fibre lasers correspond to very irregular light dynamics and a low degree of coherence. A quasi-CW fibre laser normally generates so many modes (up to $10^{6}$ ), that fluctuations in their amplitudes and phases result in a stochastic radiation, which calls for description in terms of wave turbulence ${ }^{15-19}$. To establish conditions for the existence a coherent condensate and reveal mechanisms of coherence loss, it is critically important to comprehensively study the laminar-turbulent transition in fibre laser radiation, as is done in classical hydrodynamics experiments ${ }^{2}$.

In our experiments, increasing the cavity length or the power of a fibre laser causes the output to pass from a coherent laminar state to a turbulent one. Having a laminar-turbulent transition in an optical system makes it possible to investigate fundamental questions of non-equilibrium operation in lasers: What are the mechanisms 
of losing coherence in fibre lasers? Is the transition due to an increase in temporal or spatial complexity?

The Methods section briefly describes the experimental set-up. Here we define "space" and "time" in our system to allow for meaningful comparison between fibre optics and hydrodynamics. The radiation intensity $I$ is measured in a single point as a function of time. As light makes round trips in the resonator, the radiation was measured within a series of time windows separated by the round trip time $\tau_{r t}$. The result was a function of a continuous variable within a window, denoted $t$, and a discrete variable, $T=N \times \tau_{r t}$, where $N$ is the number of round trips. The fastest process is the linear propagation with the speed of light $c$, so that $t$-dependence by the transform $t-x / c$ represents the dependence on the spatial coordinate $x$ along the resonator. The spectra of the radiation are obtained by performing a Fourier transform over $t$. Energy pumping, dissipation, dispersion and nonlinearity lead to a slow evolution of the spectra over many round trips $T$. In this way, the slow evolution coordinate $T$ has the meaning of time, while the fast time $t$ is equivalent to the longitudinal spatial coordinate $x$.

To observe the laminar-turbulent transition, the laser power was changed. The laminar regime is realized at low power and the turbulent regime at a high pump power. There is a sharp transition in the properties of the laser radiation upon the increase of the power. The optical spectrum width $\Gamma$ increases by almost twice after the power increases by only 1 percent (Fig. 1a). A sharp transition at the same power happens with the most probable intensity (Fig. 1b). Below the transition, the generation is quite stable and intensity fluctuations are small. The intensity probability density function (pdf) has a sharp narrow peak (Fig. 1b, inset) centred at the mean intensity, as it should for a coherent state. Just before the transition, the peak widens slightly, but the mean intensity remains most probable. At the transition, the most probable intensity falls by almost 
twice, while the pdf changes form and develops a wide, approximately exponential tail that manifests as a significant probability of high-intensity fluctuations. The transition is also detected as a drop in the background level of the intensity autocorrelation function from a coherent-state level to a stochastic regime (Fig. 1c). Intensity time traces just before and after the transition are shown in Fig. S3.

The transition corresponds to the loss of coherence in the system. The total number of generated modes, $M=\Gamma \times(2 L n / c) \sim 10^{5}$, so the laminar state is fundamentally different from a single-frequency (single longitudinal mode) generation ${ }^{20,21}$. Here, $n$ is the refractive index and $L$ is the fibre length. Spectrum is only twice wider after the transition and yet spatio-temporal dynamics of radiation are very different in laminar and turbulent regimes. Fig. 2 (a, b) shows rather small fluctuations before the transition, and recurring spatio-temporal patterns after the transition. We detected long-living propagating intensity minima both on a stable laminar background (Fig. 2a) and on a strongly fluctuating turbulent background (Fig. 2b). As the typical nonlinear length $L_{N L}=1 /(\gamma 1) \sim$ $1 \mathrm{~km}$ for the transition power ( $\gamma$ is a nonlinear coefficient), these structures live $\sim 100$ nonlinear lengths, so are coherent. The temporal width of the coherent structures is at the limit of our experimental resolution.

To resolve the internal details of the coherent structures observed, we used numerical modelling based on the generalized, scalar, Nonlinear Schrödinger Equation (NSE). For more details see Supplementary Information ${ }^{10}$. While the NSE is comparable to the Navier-Stokes Equation, describing the fluid flow, in terms of universality, deceptive simplicity and sheer beauty, the former is much more amenable to numerical treatment. The NSE is commonly used to describe coherent structures, stochastic-driven processes in optical fibres and fibre lasers ${ }^{11,22-27}$. 
For our fibre laser, modelling demonstrates the same laminar-turbulent transition at comparable levels of pump power. Moreover, the numerical simulations demonstrate that the laminar state is a coherent condensate, and the transition is condensate destruction. Indeed, a coherent condensate must support long acoustic waves satisfying the Bogoliubov dispersion relation, $\omega \propto k$, in distinction from usual dispersive waves with $\omega \propto k^{2}$ without the condensate ${ }^{28}$. We found that the spatio-temporal spectrum $I(k, \omega)$ had maxima along straight lines in a laminar regime and along parabolic lines after the transition (Fig. S5). Numerical simulations reveal spatio-temporal coherent structures similar to those observed experimentally (Fig. 2c, 2d). Remarkably, their shape and phase shifts are well-described by the analytical form of dark and grey solitons (Fig. S6), which are analytical solutions of the one-dimensional $\mathrm{NSE}^{29}$.

Modelling reveals the underlying mechanism of the laminar-turbulent transition in great detail and shows that the solitons are the key to the onset of turbulence. With an increase in the pump power or the cavity length, more and more solitons are generated, which leads to turbulence. Fig. 3 shows how the transition develops over the evolution coordinate $T$ at a fixed power. The moment of transition is clearly seen by spectral widening in Fig. 3a and the breakdown of spatial coherence in Fig. 3b. Solitons proliferate and cluster, creating a deep minimum that breaks the condensate (Fig. 3c-e). We concluded that the laminar-turbulent transition, observed experimentally and modelled numerically, is via the appearance, proliferation and clustering of solitons. In a linearly unstable system, solitons may appear as an outcome of instability as, for instance, for capillary-wave turbulence ${ }^{7}$.

Condensate destruction leads to creation of an intermittent state with a rather narrow spectrum, yet limited spatial coherence (along $t$ ). In contrast to the traditional (dynamic-system) view that turbulence arises from an increase in the temporal 
complexity, the spatial breakdown of coherence is the leading process here, similar to a pipe flow ${ }^{2,9}$. Even when the asymptotic turbulent stage has not yet fully developed, the condensate has already broken into pieces. When the soliton density becomes high, and the condensate is filled with dips and voids, the state is hardly distinguishable from a dense mixture of coherent bright structures (such as bright solitons and breathers). Spatiotemporal patterns expand and shrink (having a rhombic form in the $t-T$ plane) with approximately the same velocity (the slope in $\mathrm{x}$ - $\mathrm{t}$ space) as propagation of solitons on them (Fig. 2, b and d). The patterns recur quasi-periodically and move as a whole against the background, which confirms their coherent nature (Fig. S8). The intensity correlation function over the evolution coordinate shows the statistical signature of the quasiperiodical recurrence.

Repeating simulations with the only difference being a small change in the initial noise, we found that the lifetimes of the condensate fluctuate strongly, i.e. laminarturbulent transition via soliton clustering is stochastic (compare to Ref. 2). The probability to survive falls exponentially as in radioactive decay (Fig. 4). This suggests that after some time, the probability of decay is constant in time and is independent of the excitation time.

Flows are controlled by the Reynolds number, the ratio between nonlinear and linear terms in the Navier-Stokes equation. A similar ratio between nonlinearity and dispersion can be introduced and measured for a fibre laser, further developing the fluidlaser analogy (see Supplementary Information).

We observed the laminar-turbulent transition in the fibre laser radiation and identified the mechanism of such a transition, opening new possibilities for studying the fundamental problem of turbulence onset in optical devices. We discovered the critical role of coherent structures, dark and grey solitons, in destroying laser coherence, making 
a link between solitons and turbulence: localized coherent structures break the long-range coherence. A useful analogy is thus found between laser and fluid flows. Both systems can lose spatial coherence via a transition that is of a probabilistic nature. We anticipate that our results will lead to better understanding of coherence break-up in lasers and development of new optical-engineering concepts and novel classes of lasers that operate in far-from-equilibrium regimes.

\section{Methods summary}

The fibre laser used in the experiments has a standard, all-fibre design with a cavity made specifically from a high normal-dispersion fibre $(D=-44 \mathrm{ps} / \mathrm{nm} / \mathrm{km}$, nonlinear coefficient $\gamma=3 \mathrm{~km}^{-1} \mathrm{~W}^{-1}$ ) of 770 meters in length, placed between specially designed, all-fibre laser mirrors - fibre Bragg gratings. The mirrors have super-Gaussian spectral profiles of the sixth order, around $2 \mathrm{~nm}$ bandwidth, with dispersion variation of less than $10 \mathrm{ps}$ per bandwidth. This is crucial for experimental realization of the coherent laminar state and the transition to the turbulent state (see Supplementary Information for details). Fibre mirrors were written directly in a fibre core using an on-site fibre Bragg gratings writing facility, following the refractive index longitudinal profile calculated numerically to obtain the desired spectral and dispersion response.

Spatio-temporal properties of the laser radiation were analysed using an oscilloscope of $36 \mathrm{GHz}$ real-time bandwidth, comparable with optical bandwidth of the radiation. Numerical modelling was based on two complementary approaches: Analysis of longitudinal, resonator-mode evolution with round trips; and computation of field dynamics using generalized NSEs. See the Supplementary Information for details. 


\section{References}

1. Landau, L. \& Lifshits, E. Fluid Mechanics Chs. 26-28 (Pergamon Books, 1959).

2. Avila, K. et al. The onset of turbulence in pipe flow. Science 333, 192-196 (2011).

3. Siegman, A. E. Lasers (University Science Books, 1986).

4. Mandel, L. \& Wolf, E. Optical Coherence and Quantum Optics Chs. 18, 19 (Cambridge University Press, 1995).

5. Milonni P. W. \& Eberly, J. H., Laser Physics Ch. 13 (J. Wiley \& Sons, 2010).

6. Cross, M. \& Hohenberg, P. Pattern-formation outside of equilibrium. Rev. Mod. Phys. 65, 851-1123 (1993).

7. Shats, M. Parametrically excited water surface ripples as ensembles of oscillations. Phys. Rev. Lett. 108, 03452 (2012).

8. Du, Y. \& Karniadakis, G. E. Suppressing wall turbulence by means of a transverse travelling wave. Science 288, 1230-1234 (2000).

9. Hof, B., et al. Experimental observation of nonlinear travelling waves in turbulent pipe flow. Science 305, 1594-1597 (2004).

10. Agrawal, G.P. Nonlinear Fiber Optics Ch. 5 (Academic Press, 2001).

11. Turitsyna, E. G., Falkovich, G. E., Mezentsev, V. K. \& Turitsyn, S. K. Optical turbulence and spectral condensate in long-fiber lasers. Phys. Rev. A 80, 031804(R) (2009).

12. Turitsyna E. G., G. Falkovich, A. El-Taher, X., et al. Optical turbulence and spectral condensate in long fibre lasers. Proc. Royal Soc. A (2012).

13. Sun, C., et al. Observation of the kinetic condensation of classical waves. Nature Physics 8, 470-474 (2012).

14. Klaers, J., Julian Schmitt, J., Vewinger, F. \& Weitz, M. Bose-Einstein condensation of photons in an optical microcavity, Nature 468, 545-548 (2010). 
15. Shrira, V. \& Nazarenko, S. Advances in Wave Turbulence Ch. 4 (World Scientific Publishing Company, 2013).

16. Laurie, J., Bortolozzo, U., Nazarenko, S. \& Residori, S. One-dimensional optical wave turbulence: Experiment and theory, Physics Rep. 514(4), 121-175 (2012).

17. Babin, S. A., Churkin, D. V., Ismagulov, A. E., et al. FWM-induced turbulent spectral broadening in a long Raman fibre laser. J. Opt. Soc. Am. B24, 1729-1738 (2007).

18. Garnier, J., Lisak, M. \& Picozzi, A. Toward a wave turbulence formulation of statistical nonlinear optics. J. Opt. Soc. Am. B 29, 2229-2242 (2012).

19. Zakharov, V., L'vov, V. \& Falkovich, G. Kolmogorov Spectra of Turbulence Chs. 2, 3 (Springer, 1992).

20. Kringlebotn, J.T., Archambault, J.L., Reekie L. \& Payne, D.N. Er3+:Yb3+-codoped fiber distributed-feedback laser. Opt. Lett.19, 2101-2103 (1994).

21. Shi, J., Alam, S. \& Ibsen, M. Sub-watt threshold, kilohertz-linewidth Raman distributed-feedback fiber laser, Opt. Lett. 37, 1544-1546 (2012).

22. Kibler, B., et al. The Peregrine soliton in nonlinear fibre optics, Nature Physics 6, 790-795 (2010).

23. Kibler, B., et al. Observation of Kuznetsov-Ma soliton dynamics in optical fibre, Scientific Reports 2, 463 (2012).

24. Solli, D. R., Ropers, C., Koonath, P. \& Jalali, B. Optical rogue waves. Nature 450, 1054-1057 (2007).

25. Solli, D. R., Herink, G., Jalali, B. \& Ropers, C. Fluctuations and correlations in modulation instability, Nature Photonics 6, 463-468 (2012).

26. Fiber Lasers, Okhotnikov O. G., Ed. (Wiley-VCH Verlag, 2012). 
27. Churkin, D.V., Smirnov, S.V. \& Podivilov, E.V. Statistical properties of partially coherent CW fiber lasers. Optics Lett. 35, 3288-3290 (2010).

28. Bogoliubov, N.N. On the theory of superfluidity. J. Phys. (USSR) 11, 23-32 (1947).

29. Kivshar, Yu. S. \& Luther-Davies, B. Optical dark solitons: Physics and applications. Physics Rep. 298, 81-197 (1998).

Acknowledgements: We wish to thank I. Vatnik for his support at the very early stage of the experiments. This work was supported by ISF and BSF grants in Israel, ERC, Leverhulme Trust, Royal Society in the United Kingdom, and the Ministry of Science and Education and Dynasty Foundation in Russia. We thank both referees for their very helpful remarks.

Author Contributions S.K.T. and G.F. initiated the study. D.V.C. conceived the experiment and carried it out with S.S. and N.T. E.G.T. and S.V.S. designed and conducted the numerical modelling. E.G.T. and X.S. designed the special laser mirrors. X.S. fabricated the laser mirrors. G.F., S.K.T. and D.V.C. guided the theoretical and experimental investigations. G.F., S.K.T., D.V.C., E.V.P., S.A.B., S.V.S., E.G.T., S.S. and N.T. analysed data. G.F., S.K.T. and D.V.C. wrote the paper.

Competing interests' statement: The authors declare no competing financial interests.

Correspondence to: S.K. Turitsyn, e-mail: s.k.turitsyn@aston.ac.uk 


\section{Figures and captions}
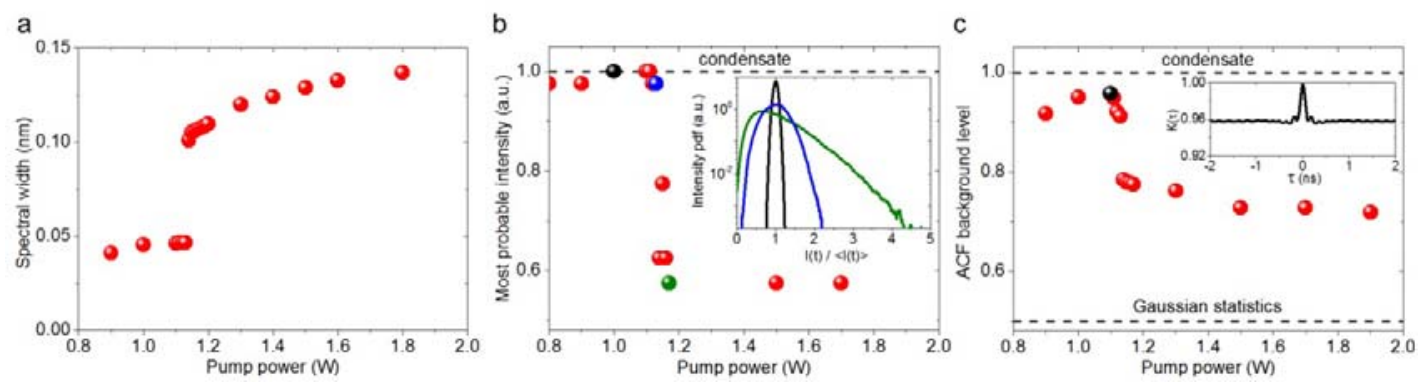

Figure 1 Laminar-turbulent transition in the fibre-laser experiment. a) The optical spectrum width (proportional to the number of excited modes) versus power. b) The most probable intensity versus power, and the full-intensity probability density functions before and after the transition (inset). The colour code attributes curves at the inset to points on the main graph. c) The background level of the intensity autocorrelation function (ACF) $K(\tau)=<I(t, T) \times I(t+\tau, T)>$ measured at large $\tau$. Inset shows typical ACF before the transition. For a coherent state, $K(\tau) \rightarrow 1$. For a completely stochastic radiation having Gaussian statistics, $K(\tau) \rightarrow 0.5$. 

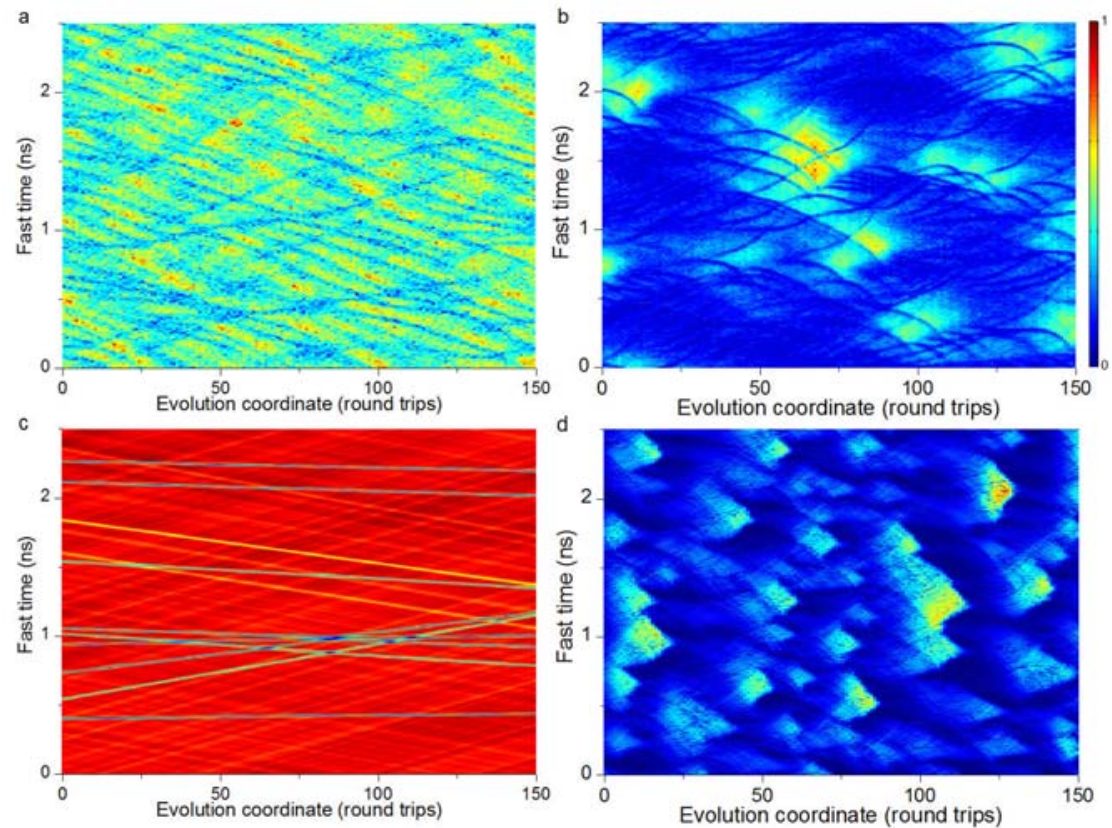

Figure 2 Coherent structures in spatio-temporal dynamics in experimental and numerical simulation in laminar and turbulent regimes. Space-time diagram of the intensity $I(t, T)$ for: a) laminar regime in experiment, b) turbulent regime in experiment, c) laminar regime in modelling, and d) turbulent regime in modelling. Evolution coordinate $T=N \times \tau_{r t}$ is shown in terms of round-trip number $N$. 

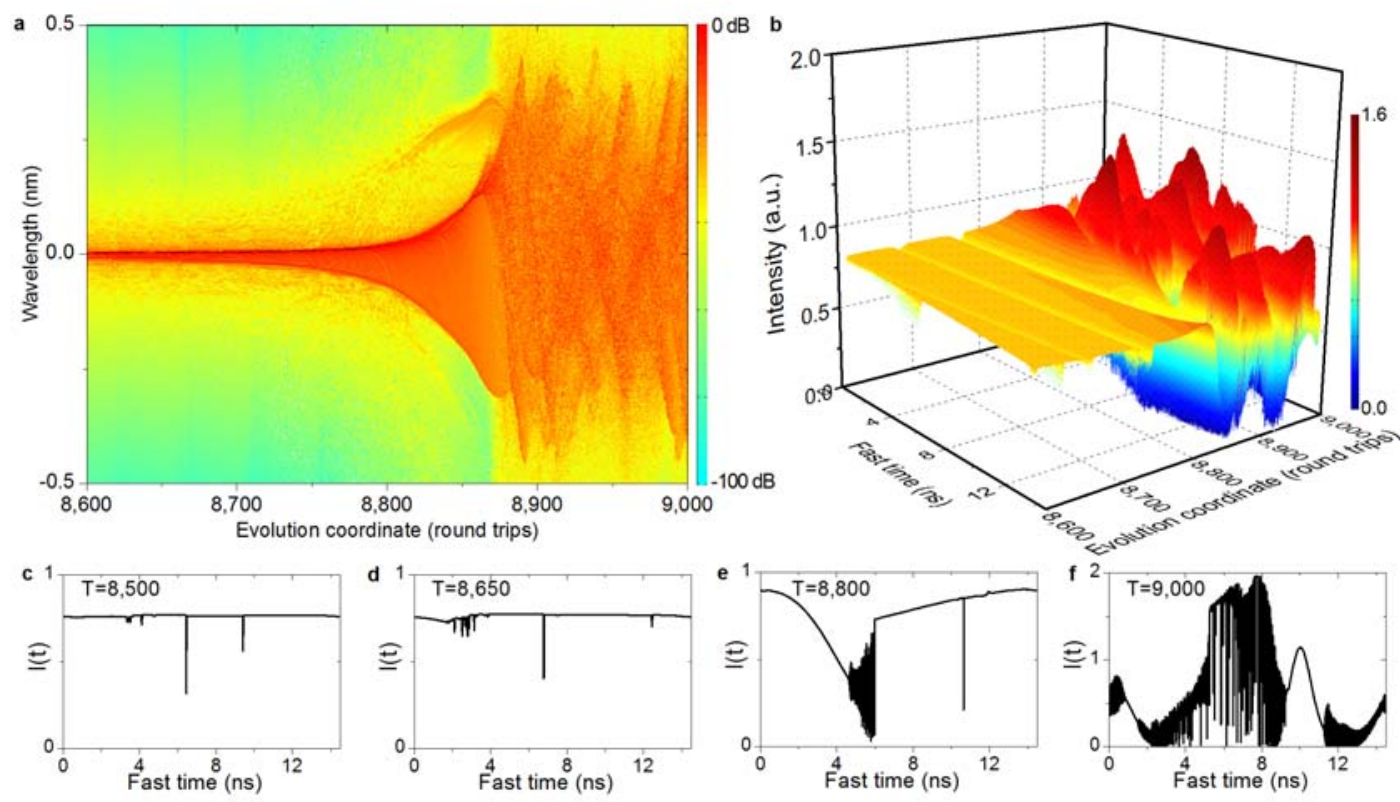

Figure 3 Soliton clustering at laminar-turbulent transition (numeric modelling) at

fixed power. a) Radiation spectrum $I(\lambda)$ in the logarithmic scale versus evolution coordinate $T$. b) Radiation intensity $I(t)$ versus $T$ : A bunch of solitons creates deepening minimum moving with a negative speed along $t$ (made into a circle; in other words, the points $t=0 \mathrm{~ns}$ and $t=2.5 \mathrm{~ns}$ are the same). At approximately $T=8,800$ round trips, this minimum is deep enough to break the condensate into two pieces, after which the total breaks down into many pieces. c-f) Radiation intensity $I(t)$ at four different $T$ : c) Condensate with rare isolated solitons, d) Beginning of soliton clustering, e) Condensate breakdown, f) Turbulence. The movie of the whole spectral and spatial evolution is shown in Video S1 in the supplementary information. Evolution coordinate $T=N \times \tau_{r t}$ is shown in terms of round-trip number $N$. 


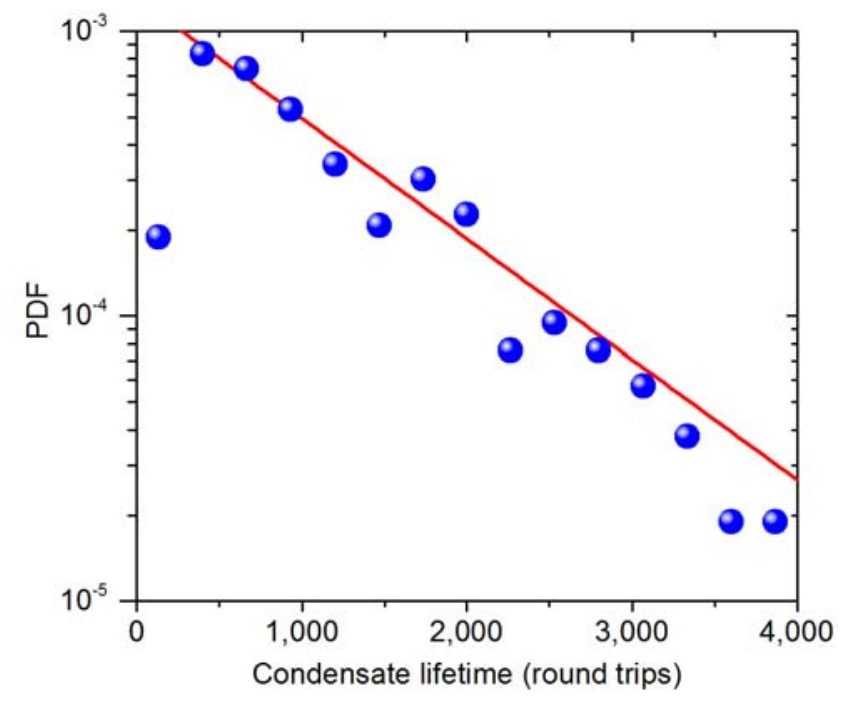

Figure 4 Probability density function for the condensate lifetime shows probabilistic nature of laminar-turbulent transition via soliton clustering. The straight line is an exponential approximation at large lifetimes. 